\title{
OPPORTUNISTIC CONTENT DISSEMINATION IN INTERMITTENT MULTI-HOP DEVICE-TO-DEVICE NETWORK
}

\author{
Yong Zhang, ${ }^{*}$ Xiaojin Zhou, ${ }^{*}$ Jin Li, ${ }^{* *}$ Xiaomei Zhang, ${ }^{* * *}$ and Mei Song*
}

\begin{abstract}
Multi-hop device-to-device (D2D) transmission can offload traffic from cellular networks. The nodes in a multi-hop D2D network cannot constantly maintain connectivity for the entire network. A hybrid network that includes integrated cellular links and intermittent multi-hop D2D links is a novel architecture. To analyse content dissemination from one publisher to multiple subscribers in this new network scenario, a propagation model is established. First, nodes must be able to receive the requested content within the timeout and successful delivery probability (SDP) constraints. The transmission probability from end to end is analysed. The relay set search algorithm (RSSA), a Dijkstra-like algorithm, is proposed to determine the node set that can receive the requested content. Based on the RSSA, the problem is to find the minimum agent set required to propagate messages to all subscribers under the timeout and SDP constraints is analysed. A solution of the set cover problem (SCP) is employed to solve this issue. Our solution is evaluated based on realistic trace data and the results verify that our solution can achieve high SDP, low delay, and low computation complexity.
\end{abstract}

\section{Key Words}

Content dissemination, multi-hop D2D network, set cover problem

\section{Introduction}

Device-to-device (D2D) systems employ a direct communication interface between pairwise user equipment (UE) to reduce the use of cellular links and improve spectrum efficiency. A lot of research has been done to analyse the performance of D2D systems, which have great advantages in capacity, delay, and power consumption [1]-[3].

* Beijing University of Posts and Telecommunications, Beijing, PR China; e-mail: yongzhang@bupt.edu.cn, zxjxwn@ sina.com, Songm@bupt.edu.cn

** Beijing Information Science \& Technology University, Beijing, PR China, e-mail: bjlijin@126.com

*** The Pennsylvania State University, University Park, PA, USA, e-mail: Xqz5057@cse.psu.edu

Corresponding author: Yong Zhang

Recommended by Dr. Xiaonv Hu

(DOI: 10.2316/Journal.206.2018.5.206-0057)
D2D communication mode is considered as a typical feature of the $5 \mathrm{G}$ mobile communication system and will be employed in future wireless networks [3]-[6].

Some researchers extend one-hop D2D mode to multihop D2D mode due to the short communication distance. The manner of direct communication between UEs in D2D systems is similar to self-organizing networks such as ad hoc and opportunistic networks (OppNets). Since 2013, multi-hop D2D technology has become a research hotspot in the D2D field, including performance analysis of the multi-hop D2D system [7]-[9], interference management [10], [11], resource allocation [12], [13], routing protocols [14]-[18], and caching technology [19], [20]. The network architecture in these works has entire network connectivity, just like mobile ad hoc networks (MANETs) or multi-hop relay networks, which is contrary to actual conditions.

As we know, it is almost impossible to maintain entire network connectivity because node density is sparse and the topology changes dynamically in the multi-hop D2D network [21]. In most cases, the architecture of a multi-hop D2D network is similar to that of an OppNet rather than a MANET [21], [22]. In a carry-store-forward approach, messages can be delivered among users without maintaining an end-to-end transmission path [23].

In this paper, the issues of content dissemination in intermittent multi-hop D2D (IMD2D) systems are investigated. IMD2D networks contain stable communication links (cellular links) and intermittent links (IMD2D links), which is a significant change in network topology. Messages can be concurrently delivered to users through cellular links. IMD2D links can offload traffic from the cellular network and improve the capacity of the entire network. Some non-real-time services, such as content dissemination services, can be offloaded to IMD2D links. Based on this new scenario, a content dissemination algorithm should be designed for this new network structure.

We investigate content dissemination from one publisher to multiple subscribers in IMD2D networks. Multihop transmission consumes a lot of cellular resources if the inband mode is adopted. To avoid the interference problem between cellular and IMD2D networks, an outband model is employed. Mobile devices are equipped with a 
cellular interface and extra wireless interfaces, such as Wi-Fi direct. Based on support from the 3GPP core network, mobile nodes know the encounter probabilities among them. A global optimization algorithm is proposed to solve the dissemination issue.

Our main contributions in this paper are described as follows:

- A novel network architecture is presented that integrates cellular links and intermittent multi-hop D2D links and a propagation model is established for content dissemination in this new scenario. The publisher can select several relay users and transmit messages to them through the cellular network while relay users transmit messages to other subscribers through IMD2D links.

- A Dijkstra-like algorithm is proposed to explore multihop paths and obtain the relay set that can receive the requested content. We also introduce a solution for the set cover problem (SCP) of finding the minimum agent set for message propagation in the IMD2D network.

- Our scheme is evaluated on real-world mobility trace data. The results show that our scheme can achieve high SDP, low delay, and low computation complexity, and effectively offload cellular traffic.

The rest of the paper is organized as follows. Section 2 reviews the existing work. Section 3 introduces the IMD2D network model. The problems concerning content dissemination in IMD2D networks are introduced. Problem formulation, including the SDP calculation, the relay set search algorithm (RSSA), and a solution for the SCP are described in Section 4. Our solution is evaluated in Section 5 using realistic trace and its performance is analysed. In the Section 6, we provide a brief conclusion.

\section{Related Work}

Some researchers have investigated multi-hop D2D networks with OppNet characteristics. Hiroki et al. analysed the requirements of multi-hop D2D networks and developed a prototype of relay by smartphone to transfer emergency messages [21]. Yunjin analysed the propagation distance in a IMD2D system [24] and discussed the size of the area affected by message dissemination at time $t$. Yong investigated the performance of the hybrid integrated cellular network, MANET, and IMD2D [25], and proposed a dynamic graph optimization framework for theoretical performance limits. Yong also proposed an optimal mobile content downloading solution in the hybrid network [26] for the transmission from many base stations to one content downloader. However, there is no existing literature discussing content dissemination from one publisher to many subscribers in an IMD2D network.

Existing methods that are designed to disseminate messages from publisher to subscribers in wireless selforganizing networks seldom take into account the effect of the cellular network. Currently, service requirements, routing protocols, buffer utilization, and so on, are the main factors in designing content dissemination algorithms in OppNet. Considering various service requirements, Cenerario et al. [27] propose a content-based dissemination protocol to exchange multi-type messages about events in a vehicle-to-vehicle network based on encounter probability. Moreover, a joint algorithm designed for routing protocol and content dissemination is an interesting research field [28]. Due to the uncertainty of node mobility, Bai et al. [29] propose a community-based single-copy forwarding protocol for DTN routing to improve the dissemination efficiency by exploiting the community structure. Some researchers also consider energy consumption; Wang et al. [30] propose a ring-based energy-aware routing algorithm for wireless sensor networks that considers the hop number, hop distance, and the residual energy of the next hop node during the routing process. Sollazzo proposes time-aware content-based dissemination system for delay tolerant networks (TACO-DTN), including content dissemination, routing protocol, and buffer management schemes [31]. Low-speed cellular links are adopted to transmit messages to special fixed infostations and then messages are delivered to the special user at a special time. So far, there is no existing content dissemination algorithm designed for the IMD2D scenario.

Due to the lack of persistent network connectivity, relay selection also attracts researchers. Using relays can improve the network coverage extension. Xiao et al. [32] propose a D2D relay-node selection scheme to select user terminals as D2D relays merely based on location information. The scheme is more feasible and can enhance data transmission in the network through multi-hop paths. Node mobility and delay tolerance were investigated in Kolios et al. [33], where D2D communications are achieved by store-carry-forward relaying under expired time constraints considering multi-hop scenarios and the assistance of base stations. Al-Hourani et al. [34] study a relayassisted D2D scenario in which the communicating devices can select the relays available in the energy-saving zone to save energy. The simulation results show that a suitable number of relays can gain substantial energy saving. Perez-Romero et al. [35] focus on the out-of-band relaying $\mathrm{D} 2 \mathrm{D}$ use case where UE can relay traffic from other UEs to the cellular infrastructure. The proposed optimization framework can determine the best connectivity option for each UE in the D2D scenario for relaying data. However, the existing research seldom considers the node mobility and some research even sets fixed relays. Our work will take node mobility into account in selecting relays.

In this paper, we will first analyse node mobility to propose a Dijkstra-like algorithm that can determine the relay node set and shortest paths. Then, a solution is introduced for the SCP of finding the minimum agent set that can cover all subscribers.

\section{Network Model}

Figure 1 illustrates the IMD2D network. The publisher owns the direct transmission link to the cellular network. The publisher can select several relay users and transmit messages to them through the cellular network. To save cellular network resources, relay users propagate these messages to other subscribers using IMD2D links. 
The following are definitions and assumptions used in this paper:

- A proximity server [1] is located in the core network and can record the neighbourhood history of mobile users. The neighbourhood history is used to predict encounter events among mobile users in the future.

- The extra second wireless interface is controlled by cellular links (i.e., controlled outband D2D communication model) or is always available (i.e., autonomous outband D2D communication model).

- Human mobility has spatial and temporal periodical properties [36]. Therefore, some parts of the mobility history can be sampled as training data. Other data can be used to verify our solution.

There are four kinds of users in the network scenario.

- Publisher: the publishers are the users who own the messages that subscribers want to get. They stably connect with the cellular network and push messages directly to relay users according to the subscriber information. The publisher selects some mobile users as relay users to forward messages to subscribers.

- Agent: agents are equipped with the cellular network interface and IMD2D interface. They receive messages from publishers through the cellular network interface and forward to subscribers through the IMD2D interface.

- Relay user: relay users are intermediate nodes between agents and subscribers that take charge of forwarding messages.

- Subscriber: subscribers are users who want to get messages from publishers. They submit subscriber information to publishers before receiving the messages they want.

The publisher pushes messages to agents through the cellular network as is shown in Fig. 1. The transmission links from the publisher to agents exist solidly. Message delivery is opportunistic in this scenario. Suppose that the successful delivery probability (SDP) constraint from agents to subscribers in time $T_{\text {lim }}$ is $P_{\text {lim }}$. Based on this

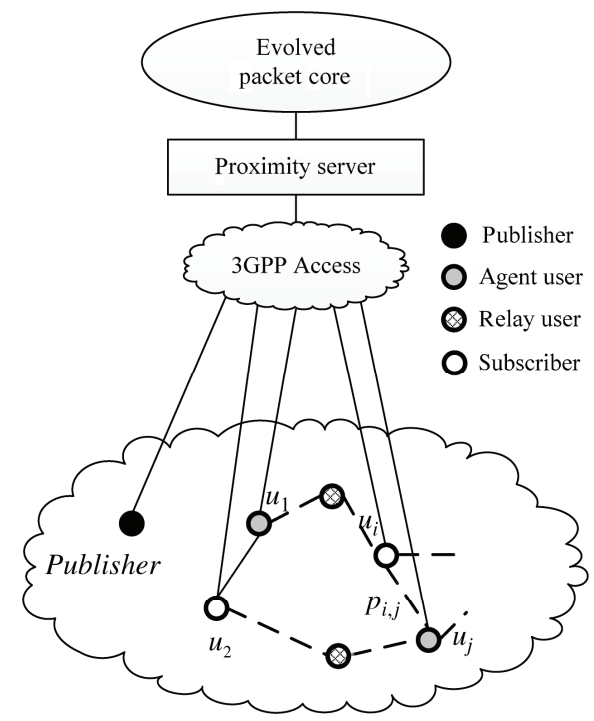

Figure 1. Network architecture.

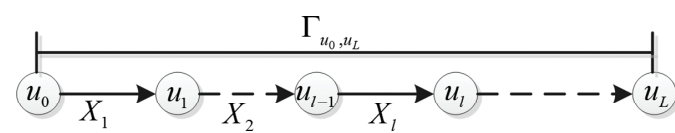

Figure 2. Opportunistic transmission path.

application scenario, there are two problems that should be solved:

Problem 1. Find the relay user set, if the agent set, timeout constraint, $T_{\mathrm{lim}}$, and $S D P$ constraint, $P_{\text {lim }}$, are given.

Problem 2. Find the minimum agent set for propagating messages to all subscribers if the timeout constraint, $T_{\mathrm{lim}}$, and $S D P$ constraint, $P_{\mathrm{lim}}$, are given.

\section{Problem Formulation}

\subsection{System Modelling}

Assume there are $N$ mobile subscribers in this network, which can be described as network graphic $G=(V, E)$. Each edge $\left(u_{i}, u_{j}\right)$ is assigned a weight $\lambda_{i, j}\left(u_{i}, u_{j} \in V,\left(u_{i}, u_{j}\right) \in E, \quad|V|=N\right)$ to represent the encounter probability. The relay node subset is $R=\left\{r_{i}\right\}$, the subscriber subset is $S=\left\{s_{i}\right\}$, and $R, S \subset V, R=V \backslash S$. $\lambda_{i, j}=\lambda_{j, i}$ due to the undirected graph. The weight graph can be represented by an adjacency matrix where

$$
w_{i, j}= \begin{cases}\lambda_{i, j}, & \text { if }\left(u_{i}, u_{j}\right) \in E \\ 0, & \text { if }\left(u_{i}, u_{j}\right) \notin E \\ 1, & \text { if } i=j\end{cases}
$$

As is shown in Fig. 2, transmission path $\Gamma_{u_{0}, u_{L}}$ from $u_{0}$ to $u_{L}$ in IMD2D can be defined as an edge set including $L$-hop links. $\Gamma_{u_{0}, u_{L}}=\left\{\left(u_{l-1}, u_{l}\right)\right\},\left\{u_{l}\right\} \subset V, l \in[0, L]$, $L \in[1, N-1]$. Wei analyses the transmission probability from $u_{0}$ to $u_{L}$ along the path $\Gamma_{u_{0}, u_{L}}$ within time $T_{\lim }$ [37]. The inter-contact time $X_{l}$ between $u_{l-1}$ and $u_{l}$ follows the exponential distribution with probability density function $p_{X_{l}}(t)=\lambda_{l-1, l} e^{-\lambda_{l-1, l} t}$. Assume an inter-contact time $X_{l}$ is independent of other edges. Transmission delay $t$ from $u_{0}$ to $u_{L}$ is the following hypo-exponential distribution:

$$
p_{T_{\text {lim }}}(t)=\sum_{k=1}^{L} C_{k}^{(L)} p_{X_{k}}(t)
$$

where the coefficients $C_{k}^{(L)}=\prod_{s=1, s \neq k}^{L} \frac{\lambda_{s-1, s}}{\lambda_{s-1, s}-\lambda_{k-1, k}}$.

The transmission path weight is

$$
\begin{aligned}
\left.p\left(T_{\lim }\right)\right|_{u_{0} \rightarrow u_{L}} & =\left.p\left(t>T_{\lim }\right)\right|_{u_{0} \rightarrow u_{L}}=\int_{T_{\lim }}^{\infty} p_{T_{\lim }}(t) d t \\
& =\sum_{k=1}^{L} C_{k}^{(L)} e^{-\lambda_{k-1, k} T_{\lim }}
\end{aligned}
$$


which indicates the timeout probability of a path $\Gamma_{u_{0}, u_{L}}$ with a length $L$ under time limitation $T_{\text {lim }}$.

Property 1: For the path $\Gamma_{u_{0}, u_{L}},\left.p\left(T_{\lim }\right)\right|_{u_{0} \rightarrow u_{L}}>$ $\left.p\left(T_{\text {lim }}\right)\right|_{u_{0} \rightarrow u_{L-1}}$.

Proof: Because $p_{u_{0}, u_{L}}\left(T_{\mathrm{lim}}\right)$ is monotonically decreasing in $T_{\text {lim }}$, let $t=t_{0, L}=t_{0, L-1}+t_{L-1, L}$,

$$
\begin{aligned}
& \left.p\left(T_{\lim }\right)\right|_{u_{0} \rightarrow u_{L}}=\left.p\left(t>T_{\lim }\right)\right|_{u_{0} \rightarrow u_{L}}=p\left(t_{0, L-1}+t_{L-1, L}>T_{\lim }\right) \\
& \quad=p\left(t_{0, L-1}>T_{\lim }-t_{L-1, L}\right)>p\left(t_{0, L-1}>T_{\lim }\right)=\left.p\left(T_{\lim }\right)\right|_{u_{0} \rightarrow u_{L-1}}
\end{aligned}
$$

Done.

Property 1 denotes that the timeout probability increases when a path grows, regardless of the length of the inter-contact time of the new link. Furthermore, the relay user set can be found using an iterative search method from agents based on Property 1.

For any node $\left(u_{0}, u_{L}\right)$, let the path $\Gamma_{u_{0}, u_{L}}$ be the path with minimum timeout probability $\left.p^{\min }\left(T_{\mathrm{lim}}\right)\right|_{u_{0} \rightarrow u_{L}}$ and length $L$. For Problem 1, the weight of transmission path $\Gamma_{u_{0}, u_{L}}$ should meet

$$
\left.p^{\min }\left(T_{\lim }\right)\right|_{u_{0} \rightarrow u_{L}} \leq p_{\lim } .
$$

First, only one node in the agent subset is considered in the following analysis. Note that our solution can easily be extended to cases in which the agent subset has more than one node. In the multi-agent case, the result is the union of the solutions of each element.

Suppose $u_{0}$ is one agent.

Lemma 1: $\forall l,\left.p^{\min }\left(T_{\lim }\right)\right|_{u_{l} \rightarrow u_{l+1}}<p_{\lim }$, if $\left.p^{\min }\left(T_{\lim }\right)\right|_{u_{0} \rightarrow u_{L}}$ $\leq p_{\lim }$ and $\Gamma_{u_{0}, u_{L}}=\left\{\left(u_{l}, u_{l+1}\right)\right\}, l \in[0, L)$.

\section{Proof:}

$$
\begin{aligned}
&\left.p^{\min }\left(T_{\lim }\right)\right|_{u_{0} \rightarrow u_{L}}=\left.p^{\min }\left(t>T_{\lim }\right)\right|_{u_{0} \rightarrow u_{L}} \\
&= p^{\min }\left(t_{0, l}+t_{l, l+1}+t_{l+1, L}>T_{\lim }\right) \\
&= p^{\min }\left(t_{l, l+1}>T_{\lim }-t_{0, l}-t_{l+1, L}\right) \\
& \because p^{\min }\left(t_{l, l+1}>T_{\lim }\right)<p^{\min }\left(t_{l, l+1}>T_{\lim }-t_{0, l}-t_{l+1, L}\right) \\
&=\left.p^{\min }\left(T_{\lim }\right)\right|_{u_{0} \rightarrow u_{L}}<p_{\lim } \\
&\left.\because p^{\min }\left(T_{\lim }\right)\right|_{u_{l} \rightarrow u_{l+1}}=p^{\min }\left(t_{l, l+1}>T_{\lim }\right)<p_{\lim }
\end{aligned}
$$

Done.

Lemma 2: $\left.p^{\min }\left(T_{\lim }\right)\right|_{u_{0} \rightarrow u_{L}}>p_{\lim }$, if $\left.p^{\min }\left(T_{\lim }\right)\right|_{u_{l} \rightarrow u_{l+1}} \geq$ $p_{\lim }$ and $\Gamma_{u_{0}, u_{L}}=\left\{\left(u_{l}, u_{l+1}\right)\right\}, l \in[0, L)$.

Proof: Based on Lemma 1, we have

$$
\left.p^{\min }\left(T_{\text {lim }}\right)\right|_{u_{0} \rightarrow u_{L}}>\left.p^{\min }\left(T_{\text {lim }}\right)\right|_{u_{l} \rightarrow u_{l+1}} \geq p_{\text {lim }} .
$$

Done.

Lemma 1 indicates that the delay of each link along the path must meet the timeout constraint requirement if

\begin{tabular}{l} 
Algorithm RSSA (undirected weighted graphic $\bar{G}$, initial node, timeout \\
constraint $T_{\text {lim }}$, SDP constraint $P_{\text {lim }}$ ) \\
\hline Output: Relay Set $R$ \\
\hline 1. Based on $\bar{G}$, assign to every node a weight value: set it to zero for the initial \\
node and to infinity for all other nodes. \\
2. Set the initial node as current. Mark all other nodes as unvisited. Create a set \\
of all the unvisited nodes called the unvisited set. \\
3. Mark the neighbour $u_{i}$ of the current node with $\left(D_{i}, R_{i}\right) . D_{i}$ is the minimum \\
weight of the path to the node $u_{0}$ according to (3). $R_{i}$ is the previous node to \\
$u_{i}$ along the shortest path. \\
4. For the current node, consider all of its unvisited neighbours and calculate \\
their weights using (3). Compare the newly calculated weights with old \\
value $D_{i}$ and select the smaller one. Mark these neighbours with the new \\
$\left(D_{i}, R_{i}\right)$. \\
5. When we are done considering all of the neighbours of the current node, mark \\
the current node as visited and remove it from the unvisited set. A visited node \\
is never checked again. \\
6. If all nodes have been marked as visited or if the smallest tentative distance \\
bctwcen the nodes in the unvisited sct and $u_{0}$ is larger than $p_{\text {lim }}$, then stop. The \\
algorithm is finished. \\
7. Select the unvisited node that is marked with the smallest tentative distance, \\
add it into relay set $R$, and set it as the new "current node," then go back to \\
Step 3 .
\end{tabular}

Figure 3. Relay set search algorithm.

the end-to-end delay is less than the timeout constraint. Lemma 2 indicates that the transmission from source to destination is excessive if the delay of any link along the path is longer than the timeout constraint. From Lemmas 1 and 2, the edges whose weights are larger than the timeout constraint can be pruned from the original graphic $G$ and subgraph $\bar{G}$, with fewer edges, is constructed.

In the following sections, a Dijkstra-like algorithm [38], called the RSSA, on $\bar{G}$ is proposed to find the relay set and the shortest path for Problem 1. Problem 2 is a typical SCP and is considered NP-hard [39]. It is difficult to find the agent set due to the computation complexity. The computation cannot be completed in limited time if we traverse all the possible combinations of pairwise nodes and find the optimal value. A greedy algorithm is employed to solve this problem [40].

\subsection{RSSA for Problem 1}

Given the pruned graphic $\bar{G}$ and agent $u_{0}$, Problem 1 becomes the task of finding the relay set whose nodes meet (4). So first, the shortest path from $u_{0}$ to other nodes and their weight should be derived. Here, the RSSA is proposed to solve this problem. The detailed description is presented as Fig. 3.

As shown in Fig. 3, RSSA is a classic Dijkstra-like algorithm. The difference is the path weight calculation method in Steps 3 and 4. Therefore, RSSA is a feasible algorithm and its time complexity is $O\left(N^{2}\right)$.

\subsection{Set Cover Problem}

The minimum SCP is known as an NP-hard problem and can be solved using integer linear programming (ILP). The SCP is stated as follows. Assume a finite set $X$ and a 
family $F$ of the subsets of $X$, so that every node in $X$ belongs to at least one subset $f$ in $F$. The goal is to find the smallest subset $C \subseteq F$ whose members cover all of $X$. Then, Problem 2 is essentially the same: find the minimum agent set $S \subseteq V$ that covers all subscribers. We will construct a covering matrix.

For any node $u_{i}$, the shortest path from $u_{i}$ to other nodes can be obtained through the RSSA. Let $N\left(u_{i}\right) \subseteq V$ be the neighbour set of the node $u_{i}$. If there exists a shortest path connecting $u_{i}$ and $u_{j}$, then $u_{j}$ is a neighbour of $u_{i}$, and all the nodes satisfying this condition form the neighbour set of $u_{i}$. Moreover, $u_{i}$ is the neighbour of itself. The neighbour set can be transformed into an $n \times n$ matrix $A$, where $a_{i j} \in\{0,1\}$ when $u_{j}$ is a neighbour of $u_{i}$. Problem 2 becomes the SCP:

$$
\min \sum_{j=1}^{n} x_{j} \quad \text { s.t. }\left\{\begin{array}{c}
A x \geq \mathbf{1} \\
\boldsymbol{x} \in\{0,1\}^{n}
\end{array}\right.
$$

A common algorithm with lower computation complexity to solve this problem is the greedy algorithm [40]. The greedy algorithm is an iterative algorithm in which nodes are selected to cover the most uncovered nodes in each iteration. It has demonstrated a logarithmic approximation ratio. A greedy algorithm can be adopted to solve Problem 2. follows:

In summary, our proposed scheme can be described as

When subscribers request a message, they submit the information request to publishers. For any node $u_{i}$, the shortest path from $u_{i}$ to other nodes can be obtained through the RSSA and the agent set that can cover all subscribers is determined by the greedy set cover algorithm. A proximity server or another network element that knows all the users' information runs these algorithms and obtains the relay set and agent set. According to the calculation results, the publisher pushes the message to agents through the cellular network and agents spread the message according to opportunistic encounters by the nodes.

\section{Evaluation and Analysis}

\subsection{Simulation Settings}

In this section, the performance of our proposed RSSA scheme is evaluated based on a realistic trace. Our evaluation is performed on the Bluetooth trace, which contains the contact history of Bluetooth devices collected by 35 devices on the campus of the University of Calabria in Rende, Italy [41]. Each device performs a periodic Bluetooth device discovery every $180 \mathrm{~s}$ to find nearby devices.

In the evaluation, all nodes request the same message and the number of agent nodes is set to 3 . We increase the constraint $T_{\lim }$ from 1 to $10 \mathrm{~min}$ and vary the minimum timeout probability $p_{\text {lim }}$ from 0.1 to 1 . In each simulation run, the first $50 \%$ of the Bluetooth trace is used as training data; other data are used to verify our solution. The process repeats 20 times for each set of timeout constraints $T_{\lim }$ and minimum timeout probabilities $p_{\mathrm{lim}}$.

\subsection{Comparison of Schemes and Evaluation Metrics}

As we know, there is no existing content dissemination algorithm designed for the IMD2D scenario. To evaluate the performance of our solution, we compare it with two other methods in the set cover procedure. We use (1) the linear programming (LP) algorithm to solve the SCP using ILP; and (2) the randomized algorithm (Random) to randomly choose agents among all of the nodes.

The metrics for evaluation are described as follows:

- Delivery ratio: this metric is the ratio of data requests being satisfied within a time constraint.

- Computing time: this metric denotes the computation complexity.

- Average delay: this metric is the average delay in getting responses to the data request.

\subsection{Evaluation Results}

\subsubsection{The Effects of the Timeout Constraint}

Figure 4 shows the impact of the timeout constraint on the system performance. It is obvious that the greedy and LP algorithms outperform the random algorithm in terms of delivery ratio and delay. The reason is that the agents selected by the greedy and LP algorithms can maximally cover the network with high SDP. This means that the greedy and LP algorithms can spread the data faster to more requesters, whereas the random algorithm may cover only a small part of the network nodes with limited propagation range and propagation speed, thereby resulting in a lower delivery ratio and longer delay.

From Fig. 4(a) and (c), we can see that when the timeout constraint increases, the delivery ratio and delivery delay of the three schemes increase correspondingly, because longer expiration implies that they can transfer more copies. Moreover, a looser timeout constraint allows nodes that require more time for successful data transmission to receive target data within the expiration time.

In addition, we compare the computing time of all three algorithms, as is shown in Fig. 4(b). Obviously, the random algorithm saves the most time because it selects three agents randomly. The greedy algorithm ranks second and its computational complexity is $O(N)$, where $N$ is the number of the nodes in the scenario. The LP algorithm is the most time consuming with a computational complexity of $O\left(N^{2}\right)$. Combining Fig. 4(a)-(c), the greedy algorithm is best for our scenario due to its ability to achieve an excellent delivery ratio and delivery delay, with little extra computational time.

\subsubsection{The Effects of Minimum Timeout Probability $p_{\text {lim }}$}

In this section, the impact of minimum timeout probability $p_{\text {lim }}$ on all schemes is analysed. From Fig. 5(a), we see that the delivery ratio of the greedy and LP algorithms increases with larger $p_{\text {lim }}$ and exceeds that of the random algorithm. As mentioned in the previous section, the greedy and LP algorithms have a high probability of transmitting data to more nodes. Furthermore, more paths are available 


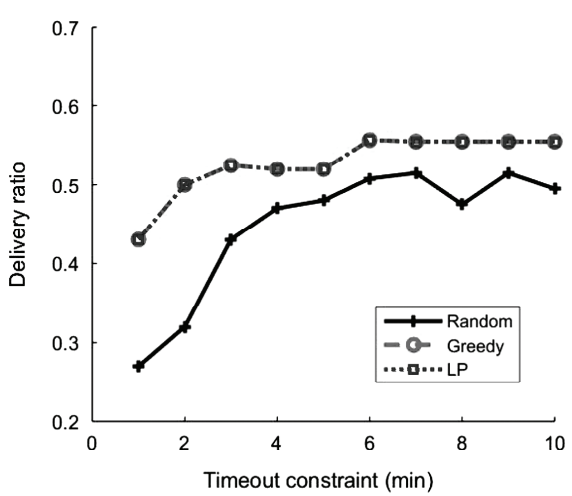

(a)

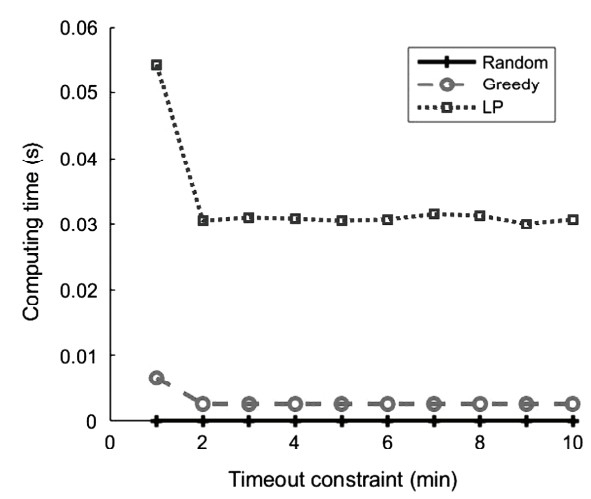

(b)

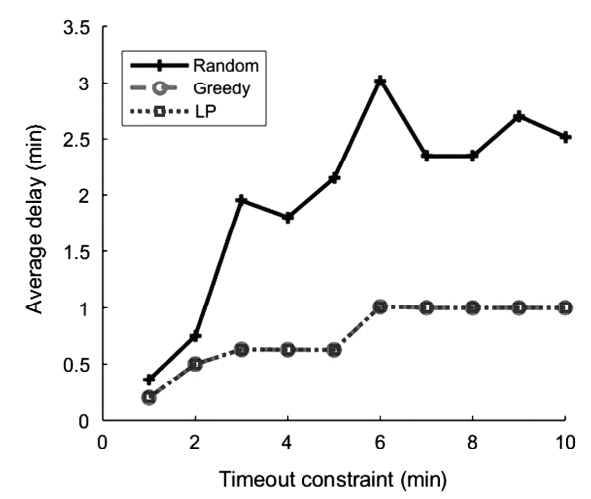

(c)

Figure 4. Performance of different timeout constraints.

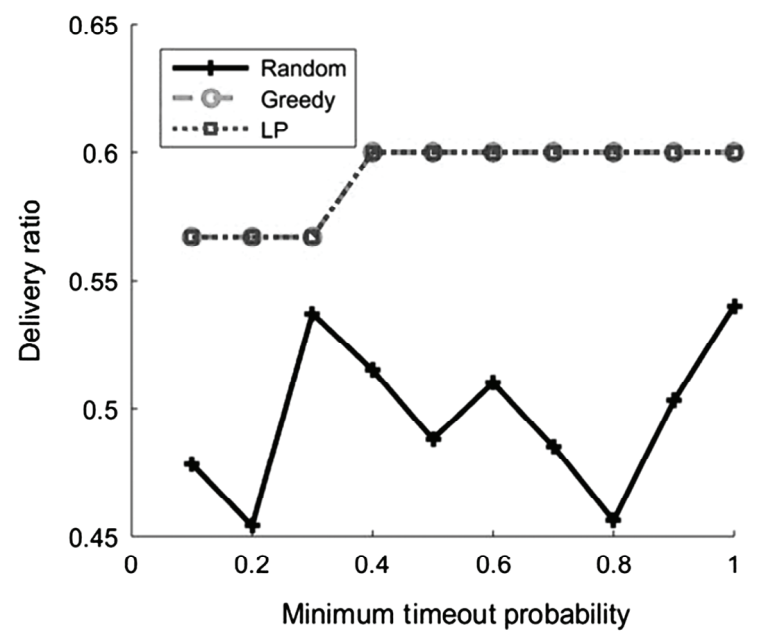

(a)

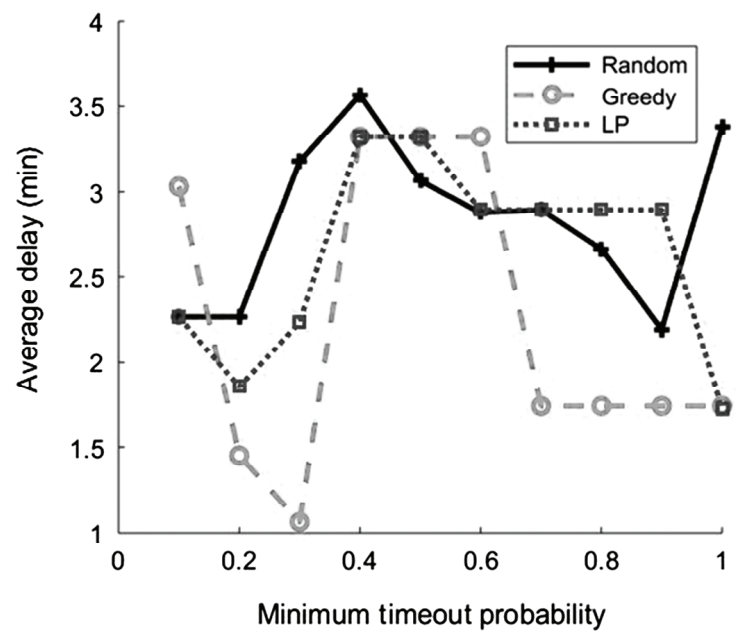

(c)

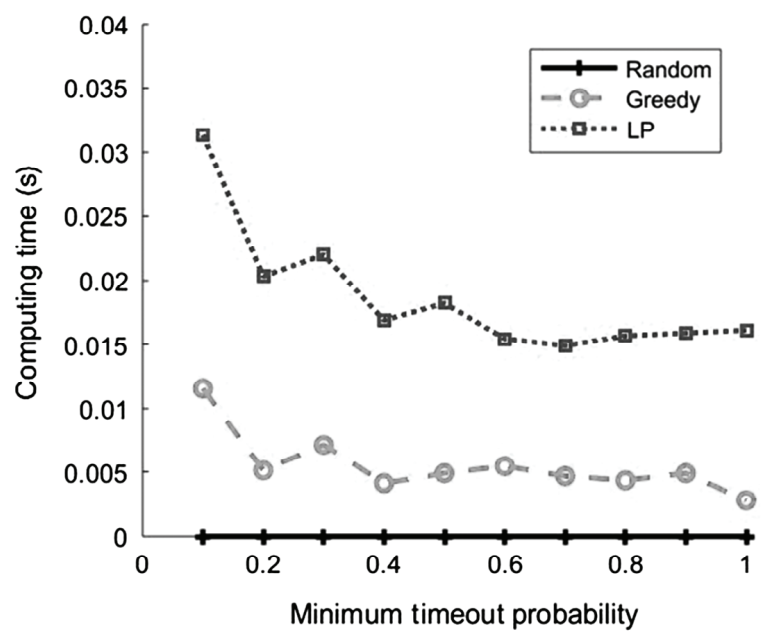

(b)

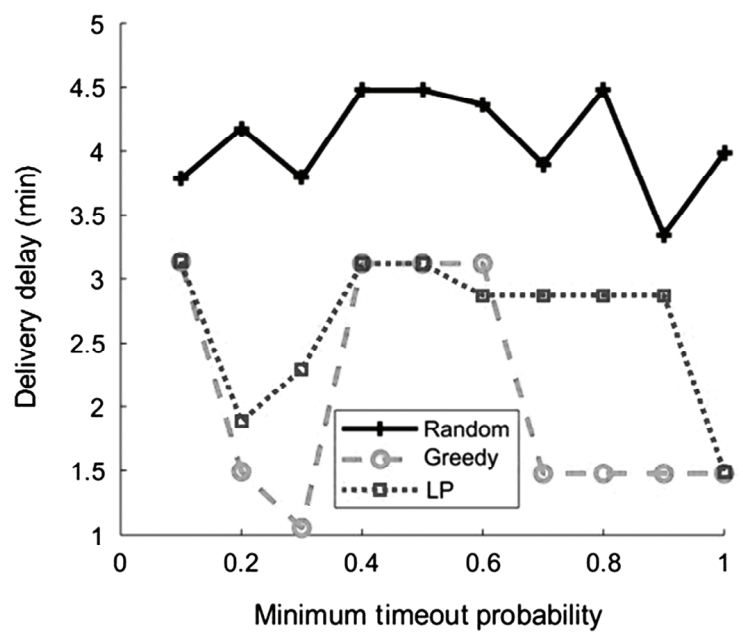

(d)

Figure 5. Performance of different minimum timeout probability $p_{\text {lim }}$.

when the minimum timeout probability $p_{\lim }$ constraint becomes looser, causing a higher probability of expanding the coverage of the selected agents.

Figure 5(c) shows that both the greedy and LP algorithms are inferior to the random algorithm concerning average delivery delay, but the result ignores the difference in the amount of the data successfully arrived according to all three algorithms. Therefore, it is necessary to sort the data according to the ascending order of the arrival time of the data at the destination node and select the time when the same number of data arrives at the destination node to compare the average delay. As shown in Fig. 5(d), the 
greedy and LP algorithms are obviously better than the random algorithm when disseminating data to the same number of nodes. As shown in Fig. 5(b), the greedy and LP algorithm computational time decreases with the minimum timeout probability $p_{\text {lim. }}$. The sparse network caused by the low minimum timeout probability $p_{\text {lim }}$ calls for more agents to cover all the nodes in the network, thereby leading to high computation complexity.

In general, the greedy algorithm is the most desirable in terms of delivery ratio and delivery delay, with little excess computational time.

\section{Conclusion}

IMD2D is a more realistic D2D network form. Core network elements make it easy to analyse the mobility characteristics of mobile nodes. The access network provides stable transmission links from the core network to mobile nodes, and the D2D link can offload traffic from the core network and improve network efficiency. The content dissemination problem in this novel network architecture is put forward and interpreted in this paper.

We proposed an SDP calculation method and the RSSA algorithm. The propagation problem is solved using a greedy algorithm and an LP algorithm to address the SCP. Real-life trace data was used to evaluate our proposal. The evaluation results show that our solution can achieve high SDP, low transmission delay, and low computation complexity.

\section{Acknowledgement}

The authors would like to thank the reviewers for their detailed reviews and constructive comments, which have helped improve the quality of this paper. This work is supported by the National Natural Science Foundation of China under Grant No. 61372117.

\section{References}

[1] K. Doppler, M. Rinne, C. Wijting, et al., Device-to-device communication as an underlay to LTE-advanced networks, IEEE Communications Magazine, 47(12), 2009, 42-29.

[2] G. Fodor, E. Dahlman, G. Mildh, et al., Design aspects of network assisted device-to-device communications, IEEE Communications Magazine, 50(3), 2012, 170-177.

[3] S. Mumtaz and R. Jonathan, Smart device to smart device communication (Cham Heidelberg, New York: Springer, 2014).

[4] M.N. Tehrani and M. Uysal, Device-to-device communication in $5 \mathrm{G}$ cellular networks challenges, solutions, and future directions, IEEE Communications Magazine, 52(5), 2014, 86-92.

[5] Q. Jian, S. Xuemin, and J. Mark, Enabling Device-to-Device Communications in Millimeter-Wave 5G Cellular Networks, IEEE Communications Magazine, 53(1), 2015, 209-215.

[6] E. Hossain, M. Rasti, H. Tabassum, and A. Abdelnasser, Evolution toward $5 \mathrm{G}$ multi-tier cellular wireless networks an interference management perspective, IEEE Wireless Communications, 21(3), 2014, 118-127.

[7] W. Peng, W. Wu, and Z.M. Li, System performance of LTEadvanced network with D2D multi-hop communication, 2013 3rd International Conf. on Consumer Electronics, Communications and Networks (CECNet), Xianning, China, 2013, 645-648.

[8] W. Si, Z. Xiaoyue, L. Yanchao, L. Zhesheng, Z. Xin, and Y. Dacheng, Achievable transmission capacity of relay-assisted device-to-device (D2D) communication underlay cellular networks, Vehicular Technology Conf. (VTC Fall), Las Vegas, USA, 2013, 1-5.

[9] L. Yong, S. Chaoming, and J. Depeng, A dynamic graph optimization framework for multihop device-to-device communication underlaying cellular networks, IEEE Wireless Communications, 21(5), 2014, 52-61.

[10] L. Babun, M. Simsek, and I. Guvenc, Intercell interference coordination for D2D discovery in LTE-A HetNets, Wireless Communications and Networking Conf. (WCNC), Istanbul, Turkey, 2014, 2202-2207.

[11] B. Kaufman, J. Lilleberg, and B. Aazhang, Spectrum sharing scheme between cellular users and ad-hoc device-to-device users, IEEE Transactions on Wireless Communications, 12(3), 2013, 1038-1049.

[12] L. Wei, R. Hu, Y. Qian, and G. Wu, Energy-efficiency and spectrum-efficiency of multi-hop device-to-device communications underlaying cellular networks, IEEE Transactions on Vehicular Technology, 47(12), 42-49.

[13] M. Hasan and E. Hossain, Resource allocation for networkintegrated device-to-device communications using smart relays, Globecom Workshops 2013, Atlanta, GA, 2013, 591-596.

[14] R. Pinyi, D. Qinghe, and S. Li, Interference-aware routing for hop-count minimization in wireless D2D networks, IEEE/CIC International Conf. on Communications in China - Workshops (CIC/ICCC), Xi'an, China, 2013, 65-70.

[15] Z. Li, Interference-aware routing and power control joint optimization in hybrid wireless networks, Applied Mechanics and Materials, 411, 2013, 856-864.

[16] H. Yuan, W. Guo, and S. Wang, Emergency route selection for D2D cellular communications during an urban terrorist attack, 2014 IEEE International Conf. on Communications Workshops (ICC), Sydney, Australia, 2014, 237-242.

[17] K. Joongheon and A.F. Molisch, Quality-aware millimeter-wave device-to-device multi-hop routing for $5 \mathrm{G}$ cellular networks, 2014 IEEE International Conf. on Communications Workshops (ICC), Sydney, Australia, 2014, 5251-5256.

[18] W. Lili, R.Q. Hu, Q.C. Li, and W. Geng, Energy-efficiency of multi-hop device-to-device communications underlaying cellular networks. 2014 IEEE International Conf. on Communications Workshops (ICC), Sydney, Australia, 2014, 5486-5491.

[19] J. Iqbal and P. Giaccone, Interest-based cooperative caching in multi-hop wireless networks, Globecom Workshops 2013, Atlanta, GA, 2013, 617-622.

[20] J. Iqbal, P. Giaccone, and C. Rossi, Local cooperative caching policies in multi-hop D2D networks, Wireless and Mobile Computing, Networking and Communications (WiMob), Larnaca, Cyprus, 2014, 245-250.

[21] H. Nishiyama, M. Ito, and N. Kato, Relay-by-smartphone: realizing multihop device-to-device communications, IEEE Communications Magazine, 52(4), 2014, 56-65.

[22] L. Yao and Y.J. Zhang, On the connectivity of underlay ad-hoc D2D networks, IEEE/CIC International Conf. on Communications in China - Workshops (CIC/ICCC), Xi'an, China, 2013, 197-203.

[23] L. Pelusi, A. Passarella, and M. Conti, Opportunistic networking: data forwarding in disconnected mobile ad hoc networks, IEEE Communications Magazine, 44(11), 2006, 134-141.

[24] L. Yujin and W. Wenye, Message dissemination in intermittently connected d2d communication networks, IEEE Transactions on Wireless Communications, 13(7), 2014, 3978-3990.

[25] L. Yong, S. Chaoming, and J. Depeng, A dynamic graph optimization framework for multihop device-to-device communication underlaying cellular networks, IEEE Wireless Communications, 21(5), 2014, 52-61.

[26] L. Yong, W. Zhaocheng, J. Depeng, and C Sheng, Optimal mobile content downloading in device-to-device communication underlaying cellular networks, IEEE Transactions on Wireless Communications, 13(7), 2014, 3596-3608.

[27] N. Cenerario, T. Delot, and S. Ilarri, A content-based dissemination protocol for VANETs: Exploiting the encounter probability, IEEE Transactions on Intelligent Transportation Systems, 12(3), 2011, 771-782.

[28] L.J. Chen, C.H. Yu, C.L. Tseng, et al., A content-centric framework for effective data dissemination in opportunistic 
networks, IEEE Journal on Selected Areas in Communications, 26(5), 2008, 761-772.

[29] B. Bai, Z. Feng, B. Zhao, and J. Su, Benefiting from the community structure in opportunistic forwarding, Computer Science and Information Systems, 10(2), 2013, 865-876.

[30] J. Wang, T. Ma, J. Cho, and S. Lee, An energy efficient and load balancing routing algorithm for wireless sensor networks, Computer Science and Information Systems, 8(4), 2011, 991-1007.

[31] G. Sollazzo, M. Musolesi, and C. Mascolo, TACO-DTN: a timeaware content-based dissemination system for delay tolerant networks, The 1st international MobiSys workshop on Mobile opportunistic networking, San Juan, Puerto Rico, 2007, 83-90.

[32] S.A. Xiao, X. Zhou, D. Feng, et al., Energy-efficient mobile association in heterogeneous networks with device-to-device communications, IEEE Transactions on Wireless Communications, 15(8), 2016, 5260-5271.

[33] P. Kolios, K. Papadaki, and V. Friderikos, Efficient cellular load balancing through mobility-enriched vehicular communications, IEEE Transactions on Intelligent Transportation Systems, 17(10), 2016, 2971-2983.

[34] A. Al-Hourani, S. Kandeepan, and E. Hossain, Relay-assisted device-to-device communication: a stochastic analysis of energy saving, IEEE Transactions on Mobile Computing, 15(12), 2016, 3129-3141.

[35] J. Perez-Romero, J. Sanchez-Gonzalez, R. Agusti, et al., Powerefficient resource allocation in a heterogeneous network with cellular and D2D capabilities, IEEE Transactions on Vehicular Technology, 65(11), 2016, 9272-9286.

[36] W.J. Hsu, T. Spyropoulos, K. Psounis, et al., Modeling spatial and temporal dependencies of user mobility in wireless mobile networks, IEEE/ACM Transactions on Networking (TON), $17(5), 2009,1564-1577$.

[37] G. Wei, et al., Cooperative caching for efficient data access in disruption tolerant networks, IEEE Transactions on Mobile Computing, 13(3), 2014, 611-625.

[38] E.W. Dijkstra, A note on two problems in connexion with graphs, Numerische Mathematik, 1(1), 1959, 269-271.

[39] M.R. Garey and D.S. Johnson, Computers and intractability: A guide to the theory of NP-completeness (Murray Hill, NJ: Bell Laboratories, Freeman Co., 1990).

[40] T. Grossman and A. Wool, Computational experience with approximation algorithms for the set covering problem, European Journal of Operational Research, 101(1), 1997, 81-92.

[41] C. Antonio, S. Annalisa, D.R. Floriano, CRAWDAD dataset unical/socialblueconn (v. 201502 08). [online]. Available: http://crawdad.org/unical/socialblueconn/20150208. (current January 2016).

\section{Biographies}

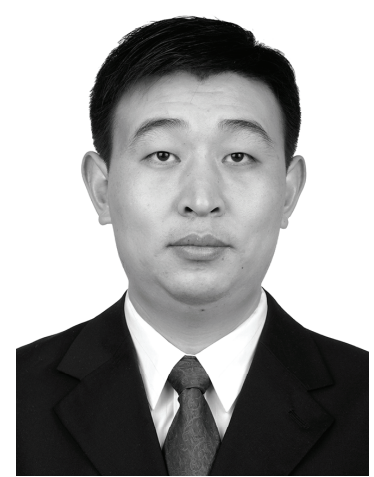

Yong Zhang has been an associate professor in the School of Electronic Engineering at Beijing University of Posts and Telecommunications (PR China). $\mathrm{He}$ holds a Ph.D. degree from Beijing University of Posts and Telecommunications. His research interests include self-organizing networks, mobile communication, and cognitive networks.

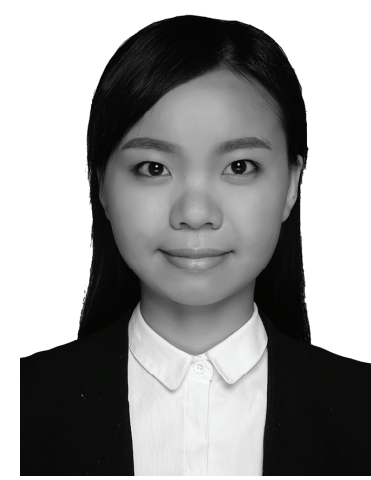

Xiaojin Zhou is an engineer in China Mobile. She holds a master degree from Beijing University of Posts and Telecommunications. Her research interests include selforganizing networks and mobile communication.

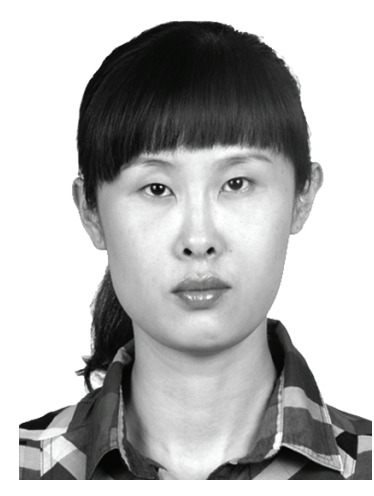

Jin $L i$ is an assistant professor at the Beijing Information Science and Technology University. She holds a master's degree from Beijing Institute of Fashion technology. Her research interests include computer-aided design.

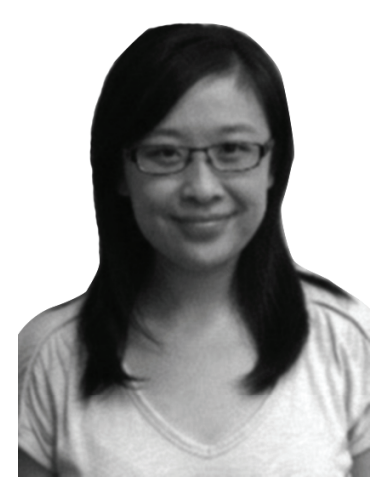

Xiaomei Zhang received the BE degree from University of Science and Technology of China in 2010. She is currently a Ph.D. candidate in the Department of Computer Science and Engineering at the Pennsylvania State University and expected to graduate in the summer of 2016. Her research interests include mobile computing, mobile social networks, data science, and machine learning.

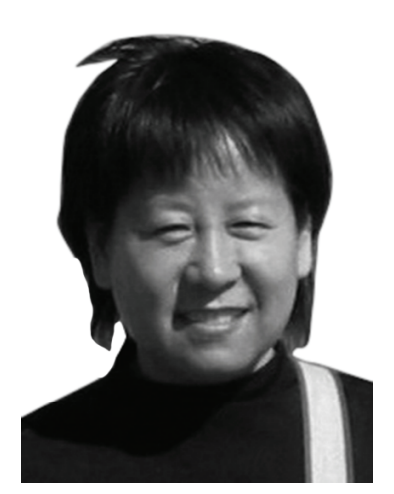

Mei Song is a Professor in Beijing University of Posts and Telecommunications. She holds a Ph.D. degree in Beijing University of Posts and Telecommunications. Her current research interests include resource allocation and mobility management in heterogeneous and cognitive network, cooperative communication, and other advanced technology in future communication. 